\title{
Contact Impedance Mapping: A New Tool to Differentiate Focal From Macroreentrant Atrial Arrhythmias
}

\author{
SUMEET K. MAINIGI, MD, FHRS and ALLAN M. GREENSPAN, MD, FACC \\ Section of Electrophysiology, Heart and Vascular Institute, Einstein Medical Center, Philadelphia, PA
}

\begin{abstract}
The aim was to evaluate a newly developed technique of active impedance mapping in differentiating arrhythmic mechanism. Differentiating focal atrial tachycardia (AT) from macroreentrant atrial flutter can sometimes be difficult, despite the use of pacing maneuvers and three-dimensional electroanatomical mapping. The variation in activation patterns which occur with focal and macroreentrant arrhythmias may be reflected in different patterns of tissue impedance. Patients undergoing supraventricular tachycardia ablation for AT or atrial flutter were included. All patients underwent a standardized electrophysiologic study and ablation procedure utilizing three-dimensional electroanatomical mapping. Impedance data were simultaneously collected. Offline analysis of impedance data was then performed. A total of 50 patients evenly split between AT and flutter were analyzed. Patients with AT were found to have a contiguous low impedance area (CLIA) in proximity to the area of successful ablation. Patients with atrial flutter had a more uniform impedance pattern with no CLIA. A subgroup of 14 patients with both AT and flutter found either during the same procedure or during subsequent procedures demonstrated a CLIA during the atrial tachycardia and uniform impedance without a CLIA during atrial flutter. Impedance mapping during atrial arrhythmias can differentiate macroreentrant atrial flutter from AT and can help localize the site of origin of an AT.
\end{abstract}

KEYWORDS. atrial flutter, atrial tachycardia, electroanatomical mapping, impedance, macroreentry, supraventricular tachycardia.

\section{Introduction}

Supraventricular tachycardia (SVT) is a common medical problem. Analysis of intracardiac electrograms and pacing maneuvers during electrophysiologic testing can nearly always differentiate the mechanism of the arrhythmia. However, focal atrial tachycardia (AT) and macroreentrant atrial flutter can often have very similar appearances of the intracardiac electrograms and response to maneuvers. In addition, the presence of significant scarring, as in patients with previous cardiac surgery or ablation, can also impede

The authors report no conflicts of interest for the published content. Manuscript received November 9, 2015, Final version accepted February 11, 2016.

Address correspondence to: Allan M. Greenspan, MD, 5501 Old York Road, Levy 3rd floor, Philadelphia, PA 19141. E-mail: greenspana@einstein.edu effective diagnosis using local activation time (LAT) mapping. Although three-dimensional LAT mapping typically shows a centrifugal atrial activation pattern in atrial tachycardias and a region where the earliest activation meets the latest activation in macroreentrant flutter, at times mapping may be misleading or inconclusive, particularly in more rapid tachycardias or in patients with more scarring.

To clarify the mechanism of these atrial arrhythmias and develop a new methodology to differentiate them, we explored the use of tissue impedance measurement during SVT. Although numerous factors can influence tissue impedance, including tissue thickness, interstitial fibrosis, scar, integrity of the lipid bilayer membrane, wall tension, and local tissue perfusion, these factors, except for blood flow and tissue thickness, do not vary with the cardiac electrical cycle, rhythm, or heart rate. 
However, an additional factor contributing to tissue impedance is the opening and closing of the membrane channels that generate the transmembrane, and perimembrane current flow. These currents vary within the cardiac electrical cycle and with heart rate. It is possible that differences in tissue impedance patterns, because of alterations in these current flows, could be detected with contact tissue impedance measurements. We hypothesize that impedance mapping might therefore allow us to reproducibly differentiate AT from atrial flutter.

\section{Methods}

\section{Patient selection}

Patients who underwent electrophysiologic study with SVT ablation at the Einstein Medical Center, Philadelphia, from March 2008 to September 2014, were evaluated. Patients found to have an SVT with a long RP interval or variable atrioventricular conduction were included in this study.

\section{Electrophysiologic study and acquisition of impedance data}

Standard electrophysiologic maneuvers were used to identify the mechanism of tachycardia. Three-dimensional electroanatomical activation mapping was performed using the Carto XP or Carto 3 mapping systems and a 4-mm tip EZ-Steer mapping catheter (Biosense-Webster, Diamond Bar, CA). While acquiring anatomical and activation data, tissue voltage and tissue impedance were collected at $87-363$ points $(176.3 \pm 63$ mean \pm SD) throughout the chamber of interest. The tissue impedance was measured by passing a $50-\mathrm{Hz}, 10-\mu \mathrm{A}$ current pulse through the ablation catheter into the tissue. Tissue voltage was measured and the tissue impedance calculated at each point and registered on the three-dimensional atrial shell. The calculated tissue impedance was based on unipolar voltage measurements between the catheter tip and the ground patch on the patient's posterior chest. Mapping point density was also calculated to ensure consistency between maps.

With no precedent in the literature for quantifying tissue impedance measurements during a tachyarrhythmia, and presumed variation in impedance measurements from patient to patient, depending on the ground patch placement, body configuration, lung volumes, etc., each patient was used as their own control. Minimum and maximum tissue impedance in the chamber of interest were measured and recorded. In a manner analogous to voltage mapping of myocardial scar, we defined an upper limit of "low" impedance as the minimum impedance plus $10 \%$ of the impedance range (maximum impedance minus minimum impedance) and defined "normal" impedance as greater than minimum impedance plus $20 \%$ of the impedance range and then dichotomized the map color scale such that red indicated "low" tissue impedance and purple indicated "normal" impedance.
All patients had definitive diagnoses made by successful radiofrequency ablation of either focal or macroreentrant atrial arrhythmias as defined by a focal site ablation or by a linear ablation transecting a critical isthmus. Ablation success was defined by the failure to reinitiate the mapped SVT with aggressive programmed atrial stimulation, including single and double atrial premature depolarizations (APD) introduced at two paced atrial cycle lengths and by burst high right atrial (HRA) and coronary sinus (CS) overdrive pacing at cycle lengths of 300-200 ms at baseline and on isoproterenol at 3-10 $\mu \mathrm{g} / \mathrm{min}$.

The impedance maps were retrospectively analyzed to determine the predicted arrhythmia mechanism and in the case of AT, the relative location of the successful ablation site and the contiguous low impedance area (CLIA).

\section{Sinus rhythm maps}

To analyze the consistency of tissue impedance measurements and characterize the impedance map in normal sinus rhythm (NSR), as a basis of comparison for the impedance maps in SVT, 14 additional patients underwent impedance maps of the right atrium in NSR and then during SVT. The mean tissue impedances during NSR and SVT were compared individually and as a group. The impedance map in NSR and the SVT were also analyzed for areas of low impedance.

\section{Statistical analysis}

Descriptive statistics were reported as mean $\pm S D$ for continuous variables that were normally distributed. Comparisons of continuous variables (impedance, cycle length, point densities, etc.) between distinct patients with atrial flutter and atrial tachycardia were evaluated using two-tailed unpaired Student t-tests. To account for the disproportionate contribution of impedance measurements from patients with multiple arrhythmias, weighted analysis was performed. To further confirm the findings, sensitivity analysis was performed that included only patients providing single sets of impedance data. Group comparisons of categorical variables were evaluated using chi-square testing. A two-tailed p-value $<0.05$ was considered statistically significant.

\section{Results}

\section{Patient demographics}

Fifty patients were included in this study equally split between patients with macroreentrant atrial flutter and AT. Table 1 outlines the demographic characteristics of the patient population. The mean age of the population was 60 years. The majority of patients $(58 \%)$ were men and similarly split between both groups. Both groups were evenly matched for coronary artery disease, hypertension, stroke, sleep apnea, diabetes mellitus type II, and ejection fraction. The mean left ventricular ejection fraction for the group was $42.9 \pm 16.7 \%$ and was similar for the two groups. 
Table 1: Patient demographics for patients with atrial tachycardia and atrial flutter.

\begin{tabular}{lccc}
\hline & Atrial flutter $(\mathbf{n}=\mathbf{2 5})$ & Atrial tachycardia $(\mathbf{n}=\mathbf{2 5})$ \\
\hline Age (years) & $60.2 \pm 10.1$ & $60.2 \pm 10.5$ & 14 \\
Gender (male) & 15 & 4 & 0.2 \\
Coronary artery disease & 4 & 16 & 0.5 \\
Hypertension & 11 & 0.5 \\
Diabetes mellitus & 8 & 5 & 0.3 \\
Hyperlipidemia & 2 & 3 & 0.5 \\
Stroke/transient ischemic attack & 2 & 3 & 0.4 \\
Obstructive sleep apnea & 1 & 3 & 0.5 \\
Ischemic cardiomyopathy & 6 & 0.6 \\
Non-ischemic cardiomyopathy & 7 & $39.7 \pm 16.8$ & 0.4 \\
LV ejection fraction (\%) & $45.8 \pm 16.1$ & 0.5 \\
\hline
\end{tabular}

CAD: coronary artery disease; CVA: cerebrovascular accident; TIA: transient ischemic attack.

\section{Evidence for the mechanism of focal atrial tachycardia}

There were 25 patients in whom 35 focal ATs were mapped and studied: 19 patients had one focal AT, four patients had two focal ATs, one patient had three focal ATs, and one had five different focal ATs. The specific mechanism of the focal AT was not directly determined in each case. All 35 focal ATs showed a centrifugal pattern of the LAT map, with the successful ablation site being at or adjacent to the site of earliest activation and with a totally negative unipolar electrogram whose onset coincided with that of the bipolar electrogram. Entrainment was employed in only seven patients, all seven of whom demonstrated long post-pacing intervals, and three focal ATs terminated with adenosine, suggesting that in these 10 instances the mechanism was triggered automaticity. All 35 ATs in the 25 patients required programmed atrial stimulation alone or in conjunction with isoproterenol for induction. Atrial overdrive pacing either reset or terminated the SVT but did not cause overdrive suppression, and none of the ATs demonstrated acceleration/deceleration behavior, arguing against enhanced automaticity as the mechanism. Finally, none of the ATs showed low amplitude $(<0.2 \mathrm{mV})$, long duration ( $>80 \mathrm{~ms})$ electrograms near the site of origin that were associated with adenosine insensitivity and were likely due to microreentry. Rather, all had electrograms that were greater than $0.5 \mathrm{mV}$ in amplitude and less than $75 \mathrm{~ms}$ in duration. In summary, these 35 focal ATs were most likely due to triggered activity or possibly microreentry.

\section{Evidence for the mechanism of atrial flutter}

The mechanism of atrial flutter was confirmed by the following observations. The LAT map demonstrated a pattern of earliest activation adjacent to the latest activation. The total activation time across the atrium accounted for greater than $80 \%$ of the atrial cycle length. Entrainment from the critical isthmus showed post-pacing intervals of less than or equal to the atrial cycle length plus $30 \mathrm{~ms}$.

\section{Variation in impedance measurements}

The mean tissue impedance for the right atrium measured during SVT was $132.3 \pm 25.3$ ohms while the mean tissue impedance for the left atrium measured during SVT was $134.5 \pm 18.4 \mathrm{ohms}(\mathrm{p}<0.01)$. The point densities for the macroreentrant SVT impedance maps averaged $1.08 \pm 0.32$ points $/ \mathrm{cm}^{2}$, while the point densities for focal AT maps averaged $1.19 \pm 0.60$ points $/ \mathrm{cm}$ $(\mathrm{p}=0.24)$ The impedances also varied between the left atrium and right atrium based on arrhythmia mechanism, and the SVT cycle length (Table 2). Weighted and sensitivity analysis confirmed these findings after accounting for patients with multiple arrhythmias.

\section{Location of focal atrial tachycardias}

Twenty-five patients underwent electrophysiologic studies with LAT and impedance mapping and were found to have 35 focal AT. Twenty-two were right ATs, 13 were left ATs.

\section{Contiguous low area of impedance}

Typical impedance maps from a right AT and a left AT with their associated LAT maps are shown in Figure 1. Both impedance maps demonstrate a CLIA, where the tissue impedance is less than or equal to the minimum impedance plus $10 \%$ of the impendence range of the entire chamber. In all 35 of the ATs, a CLIA was found in

Table 2: Variation in tachycardia cycle length and tissue impedance in right and left atria and by arrhythmia type.

\begin{tabular}{llll}
\hline & Atrial flutter & Atrial tachycardia & p \\
\hline Left atrial cycle Length & $260.0 \pm 57.5 \mathrm{~ms}$ & $349.5 \pm 136.9$ & $<0.05$ \\
Right atrial cycle Length & $251.6 \pm 35.1 \mathrm{~ms}$ & $350.5 \pm 74.7 \mathrm{~ms}$ & $<0.05$ \\
Left atrial impedance (ohms) & $138.3 \pm 12.0$ & $131.4 \pm 21.8$ & $<0.05$ \\
Right atrial impedance (ohms) & $124.9 \pm 24.6$ & $137.0 \pm 22.9$ & $<0.05$ \\
\hline
\end{tabular}




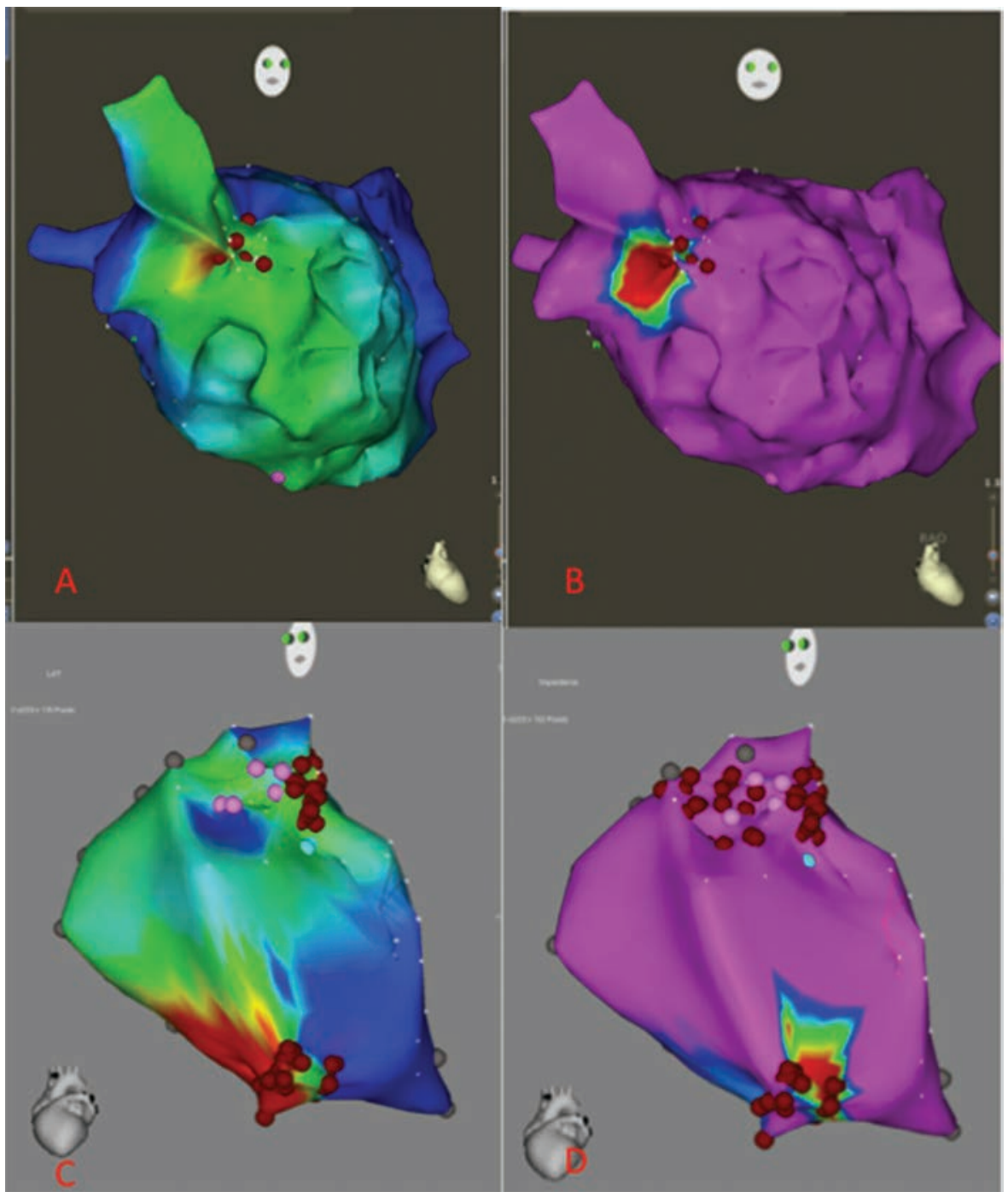

Figure 1: Corresponding activation and impedance maps of atrial tachycardias. Local activation time (LAT) maps and corresponding impedance maps for the left and right atrium are shown. (a) A LAT map of atrial tachycardia originating from below the right inferior pulmonary vein. (b) The corresponding impedance map with the area in red corresponding to the contiguous area of low of impedance. (c) A LAT map of an atrial tachycardia originating from the inferior right atrium. (d) The corresponding impedance map.

the impedance maps but was not present in any of cases of atrial flutter.

The surface area of the CLIA was measured for each AT and averaged $3.4 \pm 2.0 \mathrm{~cm}^{2}$. The measured surface area of the CLIA represented $2.4 \pm 1.8 \%$ of the atrial surface area. In each impedance map, the rest of the atrial chamber showed "normal" impedance, i.e. greater than or equal to the minimum impedance plus $20 \%$ of the impedance range.

We compared the site of origin of the AT utilizing the activation maps and successful ablation site, to the site of the CLIA. The CLIA contained the site of origin of the focal AT, i.e. the successful ablation site, in $46.4 \%$ of cases and was within $1.4 \pm 0.08 \mathrm{~cm}$ of the successful ablation site in $53.6 \%$ of the cases.

Twenty-five patients who underwent electrophysiologic studies, activation, and impedance mapping were found to have macroreentrant atrial flutters. Each patient had a successful radiofrequency ablation transecting a critical isthmus. Twenty-two of the atrial flutters were cavotricuspid isthmus dependent, two were left atrial flutters, and one was an atypical right atrial flutter. The impedance maps in all 25 patients showed almost uniform "normal" tissue impedance ( $>$ minimum impedance plus $20 \%$ of the impedance range) and no CLIA. An example of a right atrial flutter impedance map and its associated LAT map is shown in Figure 2.

Seven patients had right atrial LAT and impedance maps measured during left atrial AT. The mean right atrial impedance measured during the left atrial tachycardias was $133.6 \pm 44.2 \mathrm{ohms}$. None of the right atrial impedance maps demonstrated a CLIA during the left AT, while the left atrial impedance maps did demonstrate CLIAs. A typical example of the right and left atrial impedance maps generated during a left AT is shown in Figure 3. 

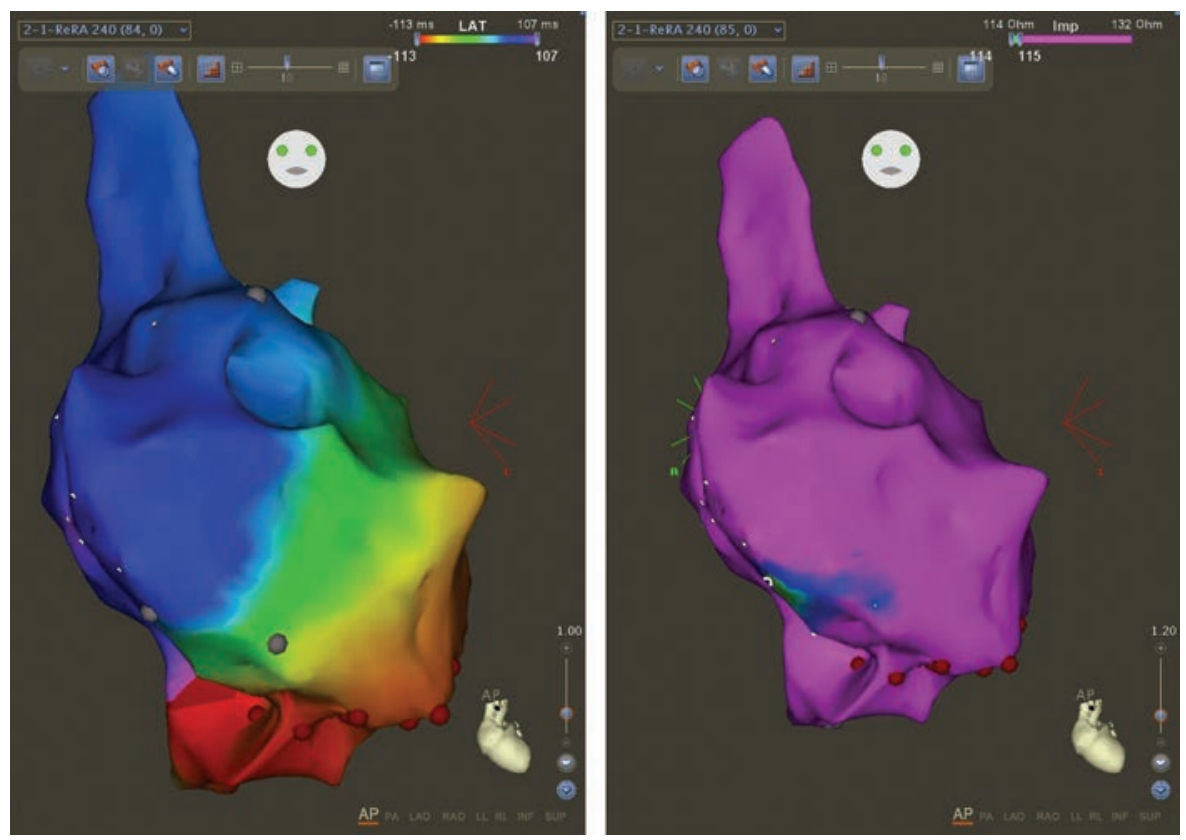

Figure 2: Corresponding activation and impedance maps of atrial flutter. The local activation timing map for a patient with typical right atrial flutter is shown on the left. On the right is the corresponding impedance map demonstrating uniform distribution of impedance.

Six of the 25 patients with inducible AT had multiple sites of origin of the tachycardia; three patients had two or three sites of AT observed the same day, while three had different sites of origin observed at different electrophysiologic studies. These six patients had a total of 16 different AT. For each AT, the impedance map demonstrated a CLIA that correlated with the focus localized by the LAT map, and successful ablation site.

Fourteen patients had both AT and atrial flutter induced: nine during the same electrophysiologic study, and five at sequential electrophysiologic studies. In each case, the impedance map during the atrial tachycardia demonstrated a CLIA adjacent to or including the site of successful ablation. In the impedance maps obtained during atrial flutter, there were no CLIA observed, and the impedance map showed a uniform pattern of "normal" impedance (Figure 4).

Histograms of the impedance distribution for the patients with AT and atrial flutter are shown in Figure 5. In atrial flutter the impedance histograms show a Gaussian distribution of the impedances for each map, while the AT impedance histograms show a characteristic skewing of the mode to lower impedances with a long tail off to the higher impedances. The difference in the distribution of atrial tissue impedances in the two arrhythmias is statistically significant $(\mathrm{p}<0.01)$

\section{Relation of scar tissue to the contiguous low impedance area}

Since scar tissue is known to have a significantly lower impedance than normal myocardium, we analyzed voltage maps obtained during the focal AT in all 25 patients to determine if the presence of scar tissue could account for the CLIA. Scar tissue, defined as atrial tissue with a voltage of
$<0.15 \mathrm{mV}$ was found in only three of the 25 patients (12\%) during the focal AT. The mean surface area of the scar tissue in the three patients was $1.37 \pm 1.2 \mathrm{~cm}^{2}$, which is smaller than the mean CLIA surface area of the CLIA $2.53 \pm 0.85 \mathrm{~cm}^{2}$ in these three patients, and substantially smaller than that of all the 25 patients, i.e. $3.4 \pm 2.0 \mathrm{~cm}^{2}$. In one patient, the scar tissue was adjacent to the successful ablation site and the CLIA, while in the other two patients the scar tissue was $>2.5 \mathrm{~cm}$ from the ablation site and the CLIA.

\section{Contact tissue impedance maps in sinus rhythm}

In an additional group of 14 patients with SVT, 11 with isthmus-dependent atrial flutter, one with atrioventricular nodal reentry tachycardia (AVNRT), one with focal septal AT, and one with a mid-cristae AT, impedance maps were obtained in both NSR before arrhythmia induction and then during SVT. Tissue impedance data were collected at 150-285 points throughout the right atrium for each map.

In six patients, five with atrial flutter (AFL) and one with the atrial septal AT, the NSR impedance map showed a uniform distribution of normal impedance without any low impedance areas. The SVT maps in these six patients showed a similar uniform distribution of normal tissue impedance in the five patients with AFL and in the one patient with the septal focal AT there was a CLIA at the site of focal AT but no low impedance area elsewhere. In the other eight patients, seven with inducible AFL and one with of the a mid-cristae AT, the NSR maps showed uniform, normal tissue impedance throughout the right atrial chamber except for a low impedance area in the posterosuperior atrial septum near the foramen ovale (FO) in seven patients and a peri-CS os CLIA in one 

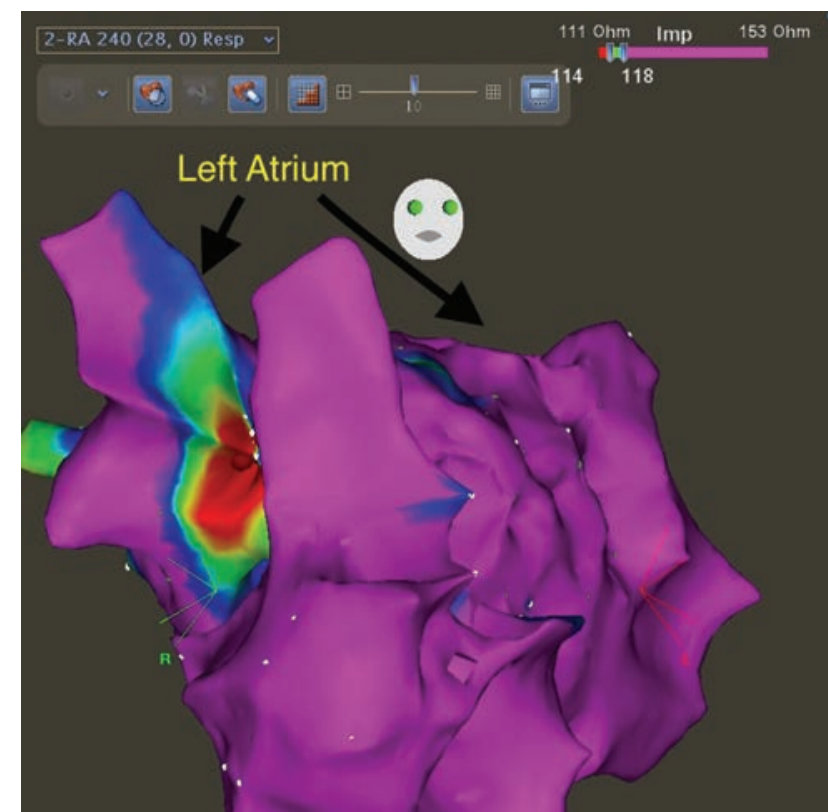

Right Atrium

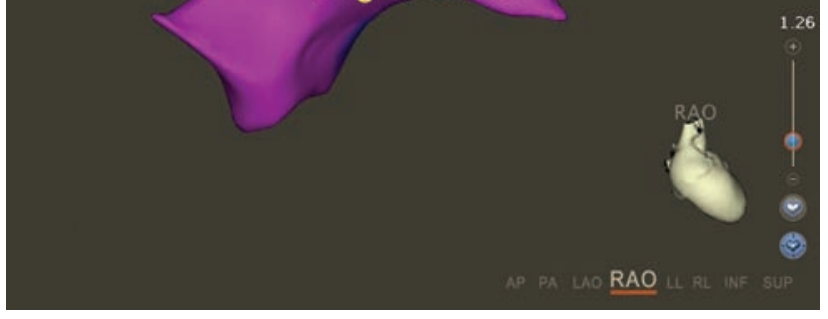

Figure 3: Biatrial impedance maps of left atrial tachycardia. Right atrial and left atrial impedance maps are shown for the patient in Figure 1 with the left atrial tachycardia. The right atrial impedance map did not demonstrate a contiguous low area of impedance while the left atrial map did which corresponded to the site of tachycardia origin.

patient. The SVT impedance maps showed the same pattern of a peri-FO CLIA only in six patients, loss of the peri-FO CLIA in one patient, and loss of the peri-CS CLIA but the appearance of the mid-cristae CLIA in the patient with the mid-cristae focal AT. An example of the NSR impedance map compared with the impedance map during atrial flutter is shown in Figure 6.

In addition we obtained sinus rhythm impedance maps in an additional 11 patients whose SVT was not inducible. These maps showed a peri-CS Os CLIA in two patients, a peri-FO CLIA in four patients and no CLIA in six patients. One patient had two CLIAs.

Overall, of the 25 patients with sinus rhythm maps, 12 patients showed uniform impedance maps, and 13 patients had CLIAs, 11 in the peri-FO region, three in the peri-CS os region (one patient having both types), but none of the sinus rhythm impedance maps showed CLIAs in the sinus node region.

\section{Discussion}

SVT is a common yet often debilitating condition. Most cases can be effectively diagnosed with electrophysiologic study and electroanatomic activation mapping, and subsequently treated with radiofrequency ablation. However, in select cases it is difficult to distinguish between AT and macroreentrant atrial flutter utilizing LAT mapping alone. As a consequence we sought a supplemental mapping strategy, using active tissue impedance measurements to aid in differentiating these arrhythmia mechanisms.

In this study we demonstrate differences in the mean active contact tissue impedance between the right atrium and left atrium. More importantly, we demonstrate that the mechanism of SVT has an impact on the pattern of the measured tissue impedance, during SVT, in each chamber. Finally, our analysis of this novel technique suggests that use of active contact impedance mapping can differentiate AT from macroreentrant atrial flutter through the identification of a contiguous low impedance area in the focal ATs that is absent in the macroreentrant atrial flutters.

\section{Rationale for the use of contact impedance mapping}

Although a number of factors may influence measured tissue impedance, regional variation in the amplitude of transmembrane and peri-membrane ionic current flows, throughout the atrial chamber during SVT could be reflected in regional variation in tissue impedance. Since $\mathrm{AT}$ and macroreentrant flutter entail regionally different patterns of transmembrane and perimembrane current flows, these differences might be expected to be demonstrable in an impedance map of the atrial chamber during the SVT.

Most focal ATs are caused by either triggered activity or microreentry, ${ }^{1}$ and either mechanism could be associated with a localized low impedance area. We reasoned that with triggered activity the current flows generated by the after-depolarizations ${ }^{2}$ that drive the membrane to threshold, particularly given the relatively large number of cells required to be synchronously activated to overcome the "source-sink" shunting of the atrial syncytium and drive the atrium, ${ }^{3}$ might be larger than those created by the longitudinal circuit current that causes transmission of the normal action potential ${ }^{4}$ throughout the rest of the atrial chamber in sinus rhythm and in macroreentrant atrial flutter. This mechanism would therefore generate a contiguous area of low impedance in atrial tachycardia. Alternatively, a micro-reentrant circuit, being continuously and rapidly activated throughout the entire cardiac cycle, and perhaps anchored by a region of scar, might also result in a localized area of lower tissue impedance, as the standard membrane channels remain open for longer periods, creating a localized higher current density. In contrast, during macroreentrant atrial flutter, tissue activation occurs via transmission of the normal action potential ${ }^{4}$ with the standard membrane ionic channels opening sequentially to generate a longitudinal circuit current that depolarizes the adjacent tissue. Thus the current flows throughout the atrial chamber during activation would be fairly uniform, as would the pattern of tissue impedance. 

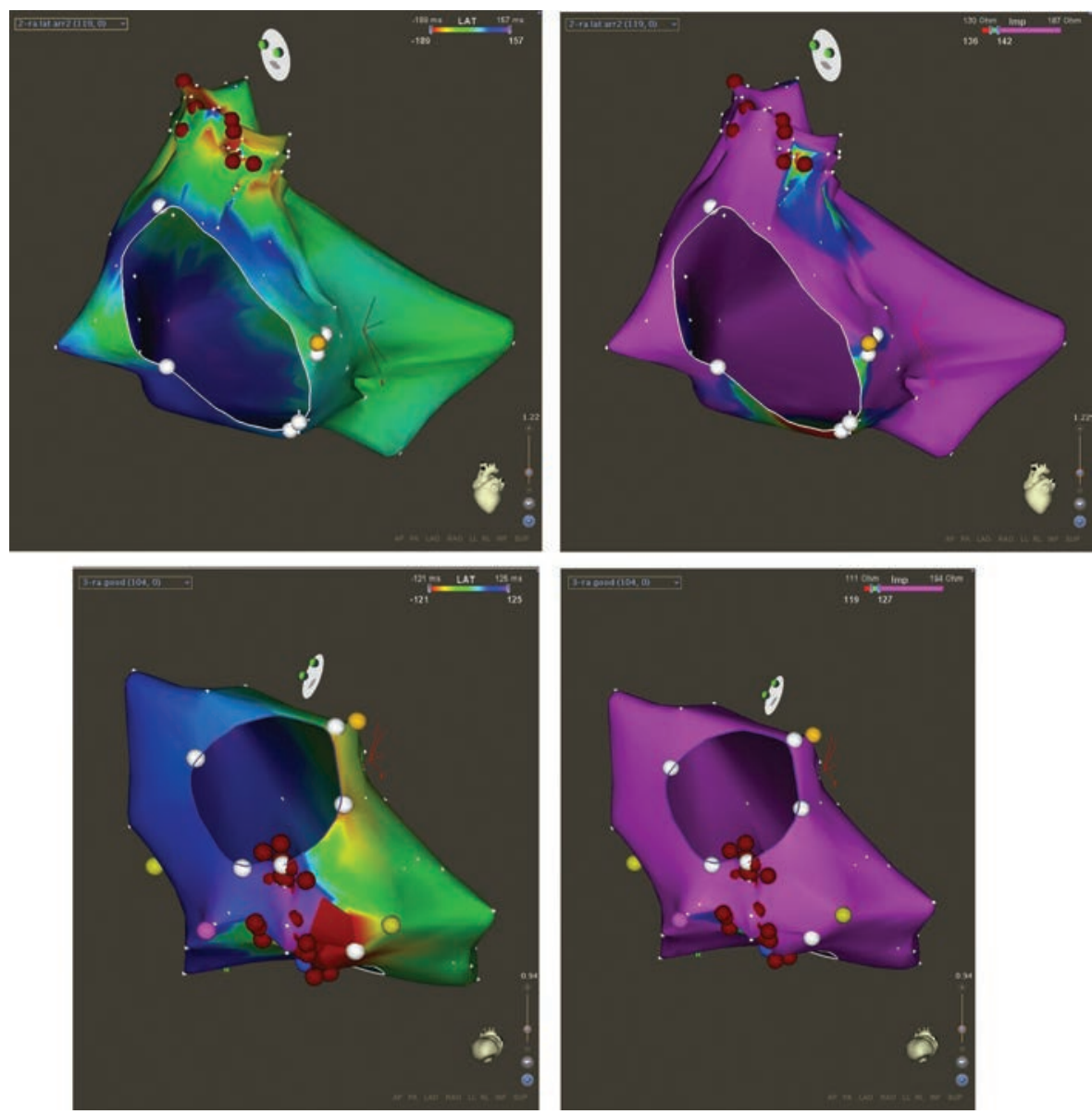

Figure 4: Corresponding activation and impedance maps for a patient with atrial tachycardia and atrial flutter. The local activation time (left panels) and impedance maps (right panels) for a patient with both and atrial tachycardia (upper panels) and cavo-tricuspid isthmus (CTI) dependent atrial flutter (lower panels) induced at the same electrophysiology study are shown. Note the contiguous area of low impedance with the atrial tachycardia (upper right) and the uniform impedance with the atrial flutter (lower right).

\section{Other factors that could generate a contiguous low impedance area}

The initial findings of differences in impedance patterns in focal AT and macroreentrant atrial flutter, which had differing atrial rates, suggested that perhaps it was a rate-related phenomenon. But this proved unlikely for several reasons.

First, the transmembrane current flows during the action potential are 2.9 times that during the resting potential. ${ }^{4}$ Because of the duty-cycle effect, at shorter atrial cycle lengths the membrane spends a larger percentage of time in the activated state than in electrical diastole and would be expected to generate a lower mean tissue impedance at shorter atrial cycle lengths. This is what is observed when comparing the mean tissue impedance during atrial flutter, which had significantly shorter atrial cycle lengths with that during focal AT. However, only the focal AT impedance map shows a confluence of lower tissue impedances in a specific location, i.e. adjacent to the successful ablation site.

The second observation that supports the fact that the CLIA is not just a rate related phenomenon is the observation that rapid atrial pacing in patients with focal $\mathrm{AT}$, at the same rate as the AT, does not generate a CLIA. This indicates that the rapid depolarizations of the atrial site by pacing involves a different mechanism from that occurring with the focal AT, particularly when the focal AT is due to triggered activity where after-depolarizations generated by multiple additional current flows drive the membrane to threshold and open the normal action potential channels.

One factor that could be responsible for the CLIA would be anatomic abnormalities such as scarring. Scar tissue is known to have a much lower tissue impedance than normal myocardium, ${ }^{5}$ and could therefore contribute to a localized low impedance area in focal AT, particularly if the mechanism is microreentry. But scarring is unlikely to be a major factor in generating the CLIA based on the following two observations. First, we have analyzed voltage maps in our 25 focal AT patients all of whom had CLIAs in their impedance maps, and only three patients $(12 \%)$ demonstrated the presence of scar. In two of these patients the scarring was remote $(>2.5 \mathrm{~cm})$ from the successful ablation site and the CLIA while in one patient it was nearby. Further, the mean scar surface area $1.37 \pm 1.2 \mathrm{~cm}^{2}$ was significantly smaller than the mean CLIA surface area of $3.4 \pm 2.0 \mathrm{~cm}^{2}$ $(\mathrm{p}<0.09)$. 

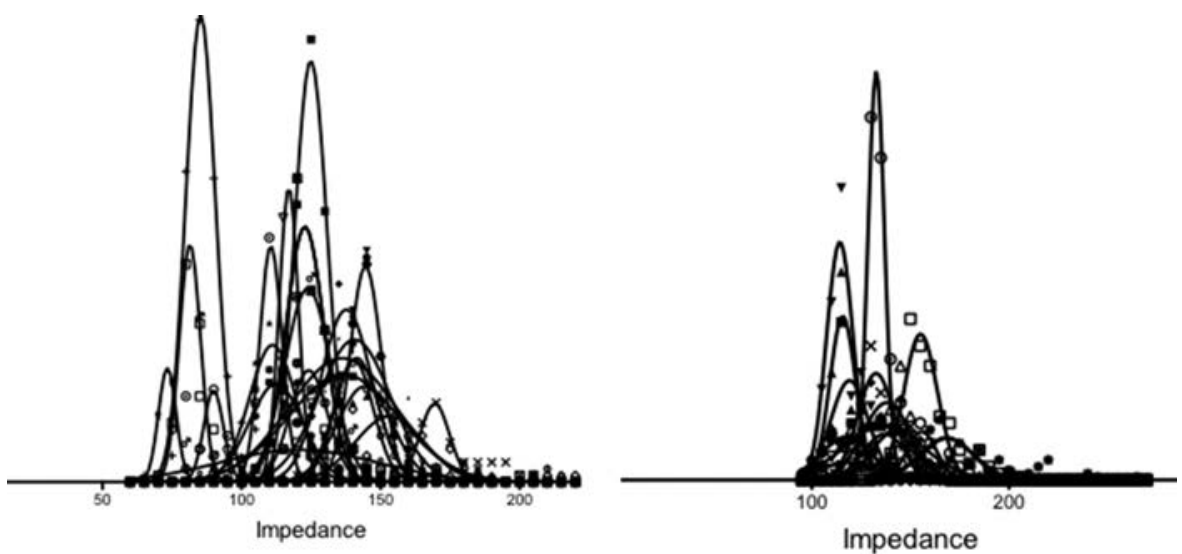

Figure 5: Impedance histograms. Frequency histograms of impedance data for individual patients are shown. On the left are patients with atrial flutter showing a Gaussian distribution. On the right are patients with atrial tachycardia showing a skew of the mode toward lower impedance.

Furthermore, we have mapped a cohort of focal AT patients with adenosine insensitivity and low amplitude $(<0.2 \mathrm{mV})$ long-duration ( $>90 \mathrm{~ms}$ ) electrograms compatible with microreentry. ${ }^{1}$ In $89 \%$ of these patients the voltage maps showed fairly large areas of scarring, most of which were adjacent to or contained the successful focal ablation site, but the impedance maps in these patients showed no CLIAs.

Finally, it is doubtful that the presence of the CLIA in focal AT maps is attributable to an anatomic abnormality, such as scar tissue. In the cohort of patients who underwent sequential ablation of focal AT and atrial flutter in the same procedure, we found the CLIA occurred only in the focal AT impedance map and not the atrial flutter map. This suggests that the CLIA reflects a physiologic phenomenon and is arrhythmia specific.

\section{Identification of a contiguous low impedance area}

Since a number of factors can produce areas of lower impedance, e.g. rapidly conducting coherent muscle bundles (e.g. Bachman's bundle) scarring and poor contact, how can we identify a CLIA indicative of a focal AT? Our

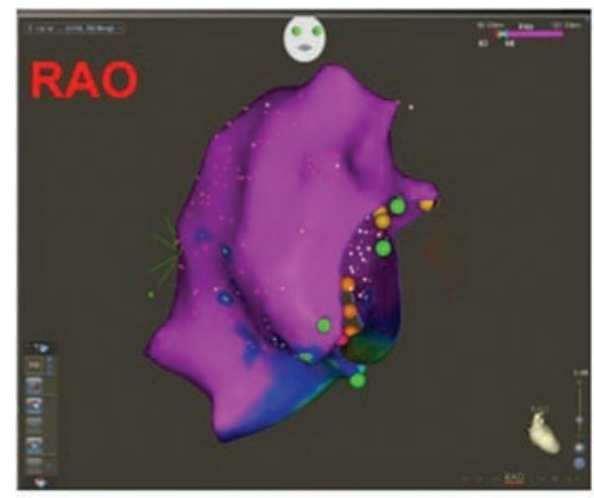

NSR observations indicate that the CLIA is found only in focal AT and not in macroreentrant atrial flutter and is of a consistent surface area. There is some evidence that the CLIA is related specifically to triggered activity as indicated by our finding of CLIAs in association with outflow tract PVCs, and not in association with adenosine insensitive focal ATs with low amplitude, long-duration electrograms ${ }^{1}$ at the ablation site. If the CLIA is specific for triggered activity, then there is an anatomic correlate for the surface area of the CLIA in the atrium, i.e. the surface area of the critical mass of contiguous atrial cells, synchronously firing, needed to overcome the "source-sink" shunting effect of the atrial syncytium. ${ }^{2,3}$ From mathematical modeling and experimental data that would be predicted to be somewhere between 300,000 to 400,000 atrial cells. Since the surface area of the average atrial cell is $3.02 \mu \mathrm{m},{ }^{6}$ the predicted surface area of a triggered focus in the atrium would be $0.9-1.2 \mathrm{~cm}^{2}$. Our finding of a consistent CLIA surface area of $3.4 \pm 2.0 \mathrm{~cm}^{2}$ is compatible with the predicted surface area, given the limitations of our measuring techniques, including issues of adequate tissue contact, and the effect of the blood pool impedance artifact that can reduce our measurement accuracy.

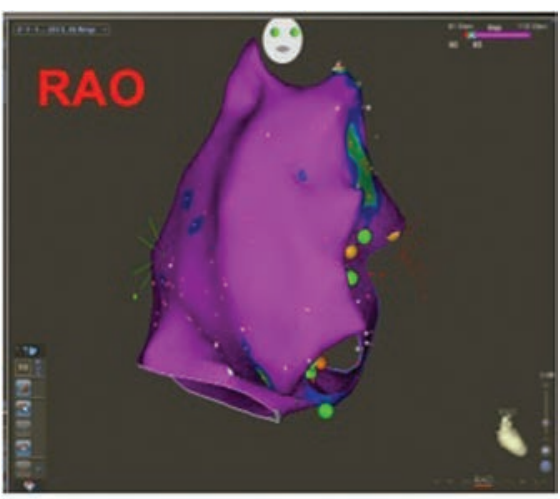

Atrial Flutter

Figure 6: Corresponding impedance maps during sinus rhythm and atrial flutter. Sinus rhythm impedance map (left panel) is compared with impedance map obtained during atrial flutter (right panel). Both impedance maps demonstrate uniform, normal tissue impedance throughout the right atrium except for a circumscribed low impedance area adjacent to the FO. 


\section{Sinus rhythm impedance maps and the lack of a low impedance area in the sinoatrial node region}

Impedance maps in sinus rhythm did not demonstrate a contiguous low impedance area in the region of the superior cristae terminalis near the sinoatrial (SA) node. This finding raises questions about our hypothesis that the CLIA may be related to increased current flows causing localized activation by either triggered activity or by microreentry. However, there are three reasons why the diastolic depolarization of SA nodal activity might not be detected as a CLIA by endocardial catheter mapping. First, the SA node is primarily an epicardial structure $^{7}$ so an endocardial catheter is not in direct contact with the region of diastolic depolarization. Second, although the current density of SA nodal cells and atrial cells are similar $(6-7 \mu \mathrm{A} / \mu \mathrm{F})$, the atrial cells are eight times larger, so the net transmembrane current for SA nodal cells is much lower, ${ }^{7}$ making detection of the contribution of the diastolic depolarization to net atrial tissue impedance more difficult. Third, diastolic depolarization is generated by only approximately 5,000 cells that constitute the "leading pacemaker site" of the SA node. ${ }^{8}$ The remaining 100,000 cells of the SA node are depolarized by local circuit current due to transmission of the action potential. This compares with the predicted number of cells in triggered activity of some 300,000400,000 necessary to overcome the "source-sink shunting effect" of the atrial syncytium and generate a focal AT. Thus the actual pacemaker current generated by diastolic depolarization is so small that it might not be sensed as an impedance change by the ablation catheter.

\section{Factors affecting tissue impedance measurements}

Because tissue impedance is being measured with a large surface area $(4 \mathrm{~mm})$ electrode of the ablation catheter a sizeable portion of the electrode is in contact with the blood pool whose impedance, being a quasi-electrolyte solution, is about half that of myocardial tissue. The impedance measurement is therefore a composite impedance (Zcom) modeled as a parallel circuit of the tissue impedance $\left(Z_{1}\right)$ and the blood pool impedance $\left(Z_{2}\right)$ given by the equation $Z c o m=Z_{1} \times Z_{2} /\left(Z_{1}+Z_{2}\right)$. Using $Z_{2}=0.5 Z_{1}$ one obtains that $Z$ com $=0.33 Z_{1}$.

The composite tissue impedance will be influenced by the volume of the blood pool because of the capacitive component of the blood pool impedance. The larger the blood pool, the lower the capacitive component of the net impedance. Thus if the mapping catheter is in the atrium and not wedged into a crevice in the atrial floor, or in a peri-annular pouch, and is freely in contact with the entire atrial blood pool, the impedance will be lower than if the electrode tip is in a small pouch, a pulmonary vein, or in the CS, where the effective blood pool volume is much smaller.

Another factor that will influence tissue impedance measurements during an arrhythmia is the preferential conduction pathway of the current and the amount of anisotropically conducting tissue involved in that pathway. For example, in the right atrium the major sources of anisotropically conducting tissue are the cristae terminalis, the trabeculated atrium, and the tendon of Todaro, all of which delimit and are excluded from the preferred conducting pathway for isthmus dependent atrial flutter. Thus the mean tissue impedance in right atrial flutter is lower than that of right atrial focal AT where part of the conducting pathway traverses these tissues. While in left atrial flutter, with four pulmonary veins orifices with fairly large net surface areas, as well as the left atrial appendage orifice and the Coumadin ridge as potential anisotropically conducting barriers, there is a lower likelihood of such a lower impedance preferential conducting pathway existing in a left atrial compared with a right atrial flutter.

\section{Future directions}

Our study demonstrates a novel observation that could potentially be a useful adjunctive technique to standard activation mapping. Further clinical and basic studies are necessary to clarify this role. Evaluation of a larger number of patients, including those with previous ablation, cardiac surgery, and congenital abnormalities where LAT mapping is less reliable because of extensive scarring will expand our understanding. To study the CLIA mechanism more fundamentally, we would propose performing experiments in an animal model to include impedance mapping in a whole animal preparation, using ionic channel blockers, to determine if the mapping system can detect changes in impedance due to changes in transmembrane current flows. Further, we propose to measure tissue impedance in a model of triggered activity and compare the impedance in the site of focal firing to that in the passively activated tissue.

\section{Limitations of study}

The major limitation of our study is the large surface area mapping catheter part of whose tip is suspended in the blood pool. This introduces a significant shunt conductance that reduces the accuracy of tissue impedance measurements. This effect of the shunt conductance could be overcome, in part by using a separate, smaller surface area mapping catheter, which however could not be used to ablate the CLIA region.

Another limitation is quantifying tissue contact with the current mapping catheters. A potential artifact of the impedance measurement could result from poor tissue contact at some sites, giving falsely low tissue impedances, thereby distorting the map. A better methodology is now available with the introduction of force sensing ablation catheters that allow quantification of tissue contact.

The third limitation is the "artifactual" CLIA introduced by the presence of coherently oriented rapidly conducting muscle bundles such as Bachman's bundle, the inferoposterior intra-atrial pathway adjacent to the CS, and those surrounding the FO. Such coherently oriented conducting bundles would also demonstrate lower than average tissue impedance reflected by rapid conduction of the electrical wave fronts. However those fiber bundles 
would show fixed anatomic areas of low impedance related to anatomic landmarks.

Finally, this was a preliminary study evaluating this novel technique in a relatively small number of patients without structural heart disease. Validation of this technique in a larger patient group, with extension to those with structural heart disease or post-ablation or postcardiac surgery is necessary, and underway.

\section{Conclusions}

We have identified a novel technique utilizing active contact impedance mapping of atrial tissue during SVT to differentiate focal atrial tachycardia from macroreentry. Our study suggests that patients with AT have an impedance map characterized by a contiguous low impedance area termed CLIA, whereas the impedance maps of macroreentrant SVT show no such low impedance area.

We have also shown that in AT, the CLIA helps to localize the successful ablation site either containing it, or being immediately adjacent to it, thereby potentially helping to focus mapping efforts in deploying the ablation lesions. Further basic and clinical studies are necessary to explore the clinical utility and improve the understanding of these findings.

\section{References}

1. Markowitz SM, Nemirovsky D, Stein KM, et al. Adenosineinsensitive focal atrial tachycardia: Evidence for de novo micro-re-entry in the human atrium. J Am Coll Cardiol. 2007; 49(12):1324-1333.

2. Weiss JN, Garfinkel A, Karagueuzian HS, Chen PS, Qu Z. Early afterdepolarizations and cardiac arrhythmias. Heart Rhythm. 2010;7(12):1891-1889.

3. Xie Y, Sato D, Garfinkel A, Qu Z, Weiss JN. So little source, so much sink: requirements for afterdepolarizations to propagate in tissue. Biophys J. 2010;99(5):1408-1415.

4. Luo CH, Rudy Y. A dynamic model of the cardiac ventricular action potential. II. Afterdepolarizations, triggered activity, and potentiation. Circ Res. 1994;74(6):1097-1113.

5. Wolf T, Gepstein L, Hayam G, et al. Three-dimensional endocardial impedance mapping: a new approach for myocardial infarction assessment. Am J Physiol Heart Circ Physiol. 2001;280(1):H179-188.

6. Spach MS, Heidlage JF, Barr RC, Dolber PC. Cell size and communication: role in structural and electrical development and remodeling of the heart. Heart Rhythm. 2004; 1(4):500-515.

7. James TN, Sherf L, Fine G, Morales AR. Comparative Ultrastructure of the sinus node in man and dog. Circulation. 1966;34(1):139-163.

8. Bleeker WK, Mackaay AJC, Masson-Pevet M, Bowman LN, Becker AE. Functional and morphologic organization of the rabbit sinus node. Circ Res. 1980;46(1):11-22. 